\title{
Joint Optimization for PS-based SWIPT Multiuser Systems with Non-linear Energy Harvesting
}

\author{
Thang X. Vu, Symeon Chatzinotas, Sumit Gautam, Eva Lagunas, and Björn Ottersten \\ Interdisciplinary Centre for Security, Reliability and Trust (SnT), University of Luxembourg. L-1855 Luxembourg. \\ E-mail: \{thang.vu, symeon.chatzinotas, sumit.gautam, eva.lagunas, bjorn.ottersten\}@uni.lu.
}

\begin{abstract}
In this paper, we investigate the performance of simultaneous wireless information and power transfer (SWIPT) multiuser systems, in which a base station serves a set of users with both information and energy simultaneously via a power splitting (PS) mechanism. To capture realistic scenarios, a nonlinear energy harvesting (EH) model is considered. In particular, we jointly design the PS factors and the beamforming vectors in order to maximize the total harvested energy, subjected to rate requirements and a total transmit power budget. To deal with the inherent non-convexity of the formulated problem, an iterative optimization algorithm is proposed based on the inner approximation method and semidefinite relaxation (SDR), whose convergence is theoretically guaranteed. Numerical results show that the proposed scheme significantly outperforms the baseline max-min based SWIPT multicast and fixed-power PS designs.
\end{abstract}

Index Terms-Simultaneous wireless information and power transfer, multiuser, energy harvesting, inner approximation, optimization.

\section{INTRODUCTION}

Next generation wireless networks focus not only on improving the spectral efficiency and ubiquitous coverage but also on green communications to improve energy efficiency. With the proliferation of mobile handsets as well as connected vehicles, this issue has even been more challenging, as the number of devices will exceed 50 billion by the end of 2020, according to Ericson's forecast [1]. These push the future communication networks to not only improve the energy management but also introduce self-powered capabilities from redundant energy sources, which is referred as energy harvesting (EH).

Among potential EH techniques, simultaneous wireless information and power transfer (SWIPT) has received much attention as a key enabling technique for future Internet-ofthing (IoT) networks. The basic premise behind SWIPT is to allow concurrent data reception and EH from the same radio frequency (RF) input signal. Restricted by the size and limited battery capacity, it has almost become essential for wireless devices to harness the potential of such techniques in order to prolong their life time. Different from the conventional receiver architectures designed for only information decoding, SWIPT requires a different architecture that allows the acquisition of both data and energy, either separately or concurrently. Two notable architectures have been proposed based on time switching (TS) and power splitting (PS) schemes [2], [3]. In the former, the received signal is switched between the information decoder and energy harvester. It is noted in the TS scheme that the full received power is used for either information decoding or energy harvesting. In the latter, both the information decoder and energy harvester are active simultaneously and each of them receives part of the signal power.

SWIPT has become a major research direction for future wireless networks recently. The authors in [4] study the rateenergy trade-off of a point-to-point system under both TS and PS mechanisms. The authors in [5] provide closed-form expressions for the cumulative distribution function (CDF) of the harvested energy and the achievable rate of EH based multiuser systems with a static PS, from which an adaptive scheduling scheme is proposed. In [6], the performance of cooperative networks with battery-powered EH relays is analyzed considering a direct source-relay link. The stochastic performance of SWIPT ad-hoc networks is studied in [7] in terms of transmission capacity and the harvested energy per unit area. The harvested energy density can be maximized by optimizing the density of the deployed information decoding nodes in TS architecture, and the power splitting ratio in PS architecture. It is noted that the above mentioned papers assume a linear EH model, which may not be consistent with practical systems.

In order to capture the saturating behavior of $\mathrm{EH}$ devices in practice [8], [9], non-linear EH models have recently been proposed [10]-[18]. In [10], a logistic function-based nonlinear EH model is proposed and applied to SWIPT systems with users requesting either information or energy. This model is employed in [11] to analyze the rate region in two-way relay channels. Another fractional-based nonlinear EH model is proposed in [12] to leverage the analysis of the harvested energy in fading channels. The rate-energy region under the nonlinear EH model is characterized in [13] for point-to-point system and in [14] for multiple-input multipleoutput broadcasting system with a single information receiver and single energy decoder. A joint user selection and power allocation scheme is proposed in [15] wherein single user is active for information decoding, while the other users only receive energy. In [16], robust beamforming design is studied to maximize the worst-case link in multi-relay networks under the nonlinear EH model. In [17], a tractable model of the rectifier non-linearity is developed to support general multicarrier modulated input waveforms, which is then used to model a wireless information and power transfer architecture based on the superposition of multi-carrier modulated and unmodulated waveforms. A comprehensive review on SWIPT is available in [18], [19]. 
TABLE I

OUR CONTRIBUTIONS COMPARED WITH RELATED WORKS

\begin{tabular}{|l|l|l|}
\hline & EH model & Performance metric \\
\hline$[20]$ & Linear & Max-min SINR \\
\hline$[21],[22]$ & Linear & Transmit power \\
\hline$[23]-[25]$ & Non-linear & Transmit power \\
\hline$[26]$ & Non-linear & Max-min received power \\
\hline This work & Non-linear & Sum harvested energy \\
\hline
\end{tabular}

\section{A. Related works}

Although research on SWIPT in multiuser systems has gained much attention, there are few works considering the joint design of the PS splitting factors and precoding vectors in SWIPT-based multiuser systems. In [20], a fairness problem is considered for multi-group multicast SWIPT systems under the linear EH model. In that work, the maximization of the minimum signal-to-noise-ratio (SINR) is formulated and solved via successive convex approximation method. The authors in [21], [22] minimize the transmit power of a multiuser system via a joint design of the precoding vectors and the receiving power factor under the linear $\mathrm{EH}$ model. A similar problem is studied in [23]-[25] under a non-linear energy harvesting model. The authors of [26] maximize the minimum received power of SWIPT networks with heterogeneous users under. It is noted that although [26] considers a non-linear EH model, their method is similar to linear EH-based designs and not applicable to maximizing the harvested energy (under non-linear EH model).

\section{B. Contributions}

In this paper, we investigate the performance of PS-based SWIPT multiuser systems in which one base station (BS) serves multiple users with information and energy simultaneously. Our goal is to jointly optimize the PS factors and the precoding vectors for every user in order to maximize the total harvested energy. Compared with [20]-[25] which either minimize the transmit power or maximize the min SINR, we maximize the total harvested energy. Compared with [26] which assumes a linear EH relation, our work is based on a realistic non-linear EH model. Table I briefly characterizes our contributions compared to related works. Particularly, a joint design of the PS factors and the precoding vectors is formulated to maximize the total harvested energy subject to users' information rate constraints. In order to overcome the inherent non-convexity of the formulated problem, we propose an iterative optimization algorithm based on the inner approximation method. The convergence of the proposed algorithm is theoretically guaranteed. The effectiveness of the proposed schemes is demonstrated via extensive numerical results, through which the impacts of key system parameters are observed. Significant EH gain is observed compared to the baseline references.

The remainder of this paper is organized as follows. Section II describes the system model and relevant variables. Section III presents the harvested energy maximization. Section IV presents numerical results. Finally, Section V concludes the paper.
Notations: $(.)^{H}$ and $\|\cdot\|$ denote the conjugate transpose operator and the $l_{2}$-norm, respectively. $\operatorname{Tr}(\boldsymbol{X})$ and $\operatorname{rank}(\boldsymbol{X})$ represent the trace and rank of matrix $\boldsymbol{X}$, respectively.

\section{SySTEM MOdEL}

We consider a SWIPT multiuser downlink in which a $N$-antenna BS serves $K$ single-antenna users via a shared wireless medium, with $K \leq N$. The set of users is denoted by $\mathcal{K}=\{1,2, \ldots, K\}$. In addition to data, the users are also interested in harvesting energy from the BS. We consider the PS mechanism [2], in which each user is equipped with an RFto-DC converter, which converts the RF power into a storable DC energy, and an information decoding module, as depicted in Fig. 1.

We consider block fading channels, in which the channel coefficients remain constant within a transmission block (or coherence time), and independently change from one block to another. Perfect channel state information (CSI) is assumed at the BS. Denote $\tilde{\boldsymbol{h}}_{k}=\sqrt{L_{k}} \boldsymbol{h}_{k} \in \mathbb{C}^{N \times 1}$ as the channel between the BS's antennas and user $k$, where $L_{k}$ is the pathloss and $\boldsymbol{h}_{k}$ is the small-scale fading channel. Before sending information to the users, the BS performs precoding to mitigate inter-user interference. Let $s_{k}$ and $\boldsymbol{w}_{k} \in \mathbb{C}^{N \times 1}$ denote the data symbol and precoding vector for user $k$, respectively. The received signal at user $k$ is given as:

$$
y_{k}=\sum_{l=1}^{K} \tilde{\boldsymbol{h}}_{k}^{H} \boldsymbol{w}_{l} s_{l}+n_{A, k}
$$

where $n_{A, k}$ is the Gaussian noise at the user $k$ 's receive antenna with zero mean and variance $\sigma_{A}^{2}$.

\section{A. Energy harvesting model}

We employ the PS mechanism to harvest energy [2], in which the received signal's power is split for information decoding and energy harvesting. The joint design of the PS

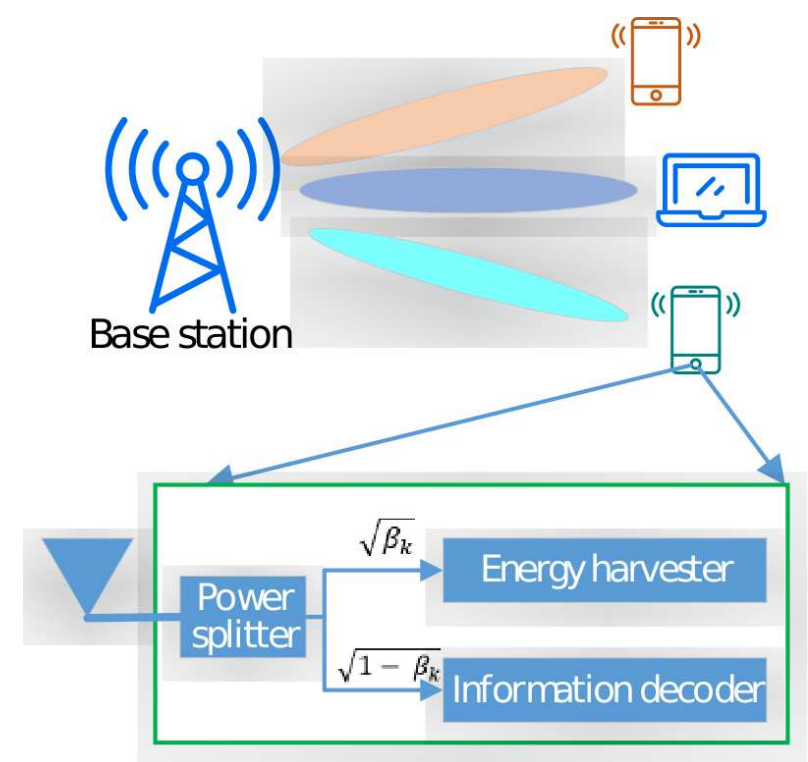

Fig. 1. Simultaneous wireless information and power transfer multiuser system with the power-splitting architecture. 
factors is carried out by the BS which then notifies the users of the optimal PS factors via, e.g., reliable feedback channel. The power input to user $k$ 's $\mathrm{EH}$ module is given as

$$
P_{R, k}=\beta_{k}\left(\sum_{m} L_{k}\left|\boldsymbol{h}_{k}^{H} \boldsymbol{w}_{m}\right|^{2}+\sigma_{A}^{2}\right),
$$

where $0 \leq \beta_{k} \leq 1$ denotes the PS fraction used for harvesting energy.

We consider practical non-linear EH model [10], [23]-[25], in which the harvested energy is non-linearly dependent on the input power ${ }^{1}$. Specifically, the harvested energy at user $k$ is given as

$$
E_{k}\left(P_{R, k}\right)=\frac{E_{\max }}{1-c}\left(\frac{1}{1+\exp \left(-a P_{R, k}+a b\right)}-c\right),
$$

where $P_{R, k}$ is given in (2), $E_{\max }$ is the maximum harvested energy of the circuit at the saturated point, $a, b$ are constants depending on the circuit which are usually obtained by fitting with the measured $\mathrm{data}^{2}$, and $c=\frac{1}{1+\exp (a b)}$.

\section{B. Information decoding model}

The received signal at the information decoding module of user $k$ is given as

$$
\begin{aligned}
& y_{I, k}=\sqrt{1-\beta_{k}}\left(\sum_{l=1}^{K} \tilde{\boldsymbol{h}}_{k}^{H} \boldsymbol{w}_{l} s_{l}+n_{A, k}\right)+n_{I, k} \\
& =\sqrt{1-\beta_{k}} \tilde{\boldsymbol{h}}_{k}^{H} \boldsymbol{w}_{k} s_{k}+\sqrt{1-\beta_{k}}\left(\sum_{l \neq k} \tilde{\boldsymbol{h}}_{k}^{H} \boldsymbol{w}_{l} s_{l}+n_{A, k}\right)+n_{I, k},
\end{aligned}
$$

where $n_{I, k}$ is the Gaussian noise introduced by user $k$ 's power splitter with zero mean and variance $\sigma_{I}^{2}$ [2]. In (4), the first term is the desired signal for user $k$, and the second term is aggregated inter-user interference due to concurrent transmissions. The achievable information rate for user $k$ is given as:

$$
R_{k}=B \log _{2}\left(1+\frac{\left(1-\beta_{k}\right) L_{k}\left|\boldsymbol{h}_{k}^{H} \boldsymbol{w}_{k}\right|^{2}}{\left(1-\beta_{k}\right)\left(\sum_{l \neq k} L_{k}\left|\boldsymbol{h}_{k}^{H} \boldsymbol{w}_{l}\right|^{2}+\sigma_{A}^{2}\right)+\sigma_{I}^{2}}\right),
$$

where $B$ is the channel bandwidth.

\section{MAXIMIZATION OF HARVESTED ENERGY}

In this section, we aim to maximize the sum harvested energy from all users, with each user subjected to a qualityof-service $(\mathrm{QoS})$ requirement of the information rate. In particular, we jointly design the beamforming vectors $\boldsymbol{w}_{k}$ and the power splitting factors $\beta_{k}$ under limited total transmit power. The optimization problem is formulated as follows:

$$
\begin{array}{cc}
\underset{\boldsymbol{w}_{k}, \beta_{k}}{\operatorname{maximize}} \sum_{k=1}^{K} E_{k}\left(\beta_{k}\left(\sum_{i=1}^{K} L_{k}\left|\boldsymbol{h}_{k}^{H} \boldsymbol{w}_{i}\right|^{2}+\sigma_{A}^{2}\right)\right) \\
\text { s.t. } & \frac{\left(1-\beta_{k}\right) L_{k}\left|\boldsymbol{h}_{k}^{H} \boldsymbol{w}_{k}\right|^{2}}{\left(1-\beta_{k}\right)\left(\sum_{l \neq k} L_{k}\left|\boldsymbol{h}_{k}^{H} \boldsymbol{w}_{l}\right|^{2}+\sigma_{A}^{2}\right)+\sigma_{I}^{2}} \geq \bar{\eta}_{k}^{R}, \forall k \\
& \sum_{k=1}^{K} \boldsymbol{w}_{k}^{H} \boldsymbol{w}_{k} \leq P_{t o t} ; 0 \leq \beta_{k} \leq 1, \forall k,
\end{array}
$$

\footnotetext{
${ }^{1} \mathrm{We}$ assume that the received power at the $\mathrm{EH}$ module is above a minimum threshold that effective energy can be harvested.

${ }^{2}$ These parameters might vary depending on the near-field or far-field measurements.
}

where $E_{k}(x)$ is given in (3), $\bar{\eta}_{k}^{R}=2^{\eta_{k}^{R} / B}-1, \eta_{k}^{R}$ is the minimum rate requirement of user $k$, and the last constraint is to satisfy the total transmit power $P_{t o t}$ at the BS.

Solving problem (6) is challenging due to the non-convexity of the received power (with respect to both $\beta_{k}$ and $\boldsymbol{w}_{k}$ ) as well as the non-convexity of function $E_{k}(x)$ (in fact it is either convex or concave depending on the parameters). To overcome this challenge, we employ new variables $\boldsymbol{W}_{k}=\boldsymbol{w}_{k} \boldsymbol{w}_{k}^{H}$ and denote $\boldsymbol{H}_{k}=\boldsymbol{h}_{k} \boldsymbol{h}_{k}^{H}$. It is evident that $\left|\boldsymbol{h}_{k}^{H} \boldsymbol{w}_{i}\right|^{2}=$ $\operatorname{Tr}\left(\boldsymbol{H}_{k} \boldsymbol{W}_{i}\right)$ and $\boldsymbol{w}_{k}^{H} \boldsymbol{w}_{k}=\operatorname{Tr}\left(\boldsymbol{W}_{k}\right)$. Then by introducing slack positive variables $\left\{x_{k}, y_{k}, z_{k}\right\}_{k=1}^{K}$, problem (6) can be reformulated as follows:

$$
\begin{aligned}
& \underset{\substack{\operatorname{maximize} \\
\boldsymbol{W}_{k}, \beta_{k}, x_{k}, y_{k}, z_{k}}}{ } \frac{E_{\max }}{1-c} \sum_{k=1}^{K}\left(x_{k}-c\right) \\
\text { s.t. } & e^{y_{k}}+1 \leq \frac{1}{x_{k}}, \forall k \\
& a \beta_{k} z_{k}+y_{k} \geq a b, \forall k, \\
& \sum_{i=1}^{K} L_{k} \operatorname{Tr}\left(\boldsymbol{H}_{k} \boldsymbol{W}_{i}\right)+\sigma_{A}^{2} \geq z_{k}, \forall k \\
\frac{\operatorname{Tr}\left(\boldsymbol{H}_{k} \boldsymbol{W}_{k}\right)}{\bar{\eta}_{k}^{R} \geq} & \sum_{l \neq k} \operatorname{Tr}\left(\boldsymbol{H}_{k} \boldsymbol{W}_{l}\right)+\frac{\sigma_{A}^{2}}{L_{k}}+\frac{\sigma_{I}^{2}}{L_{k}\left(1-\beta_{k}\right)}, \forall k, \\
& \sum_{k=1}^{K} \operatorname{Tr}\left(\boldsymbol{W}_{k}\right) \leq P_{t o t}, \\
& \boldsymbol{W}_{k} \succeq 0 ; \operatorname{rank}\left(\boldsymbol{W}_{k}\right)=1, \forall k .
\end{aligned}
$$

In (7), constraints (7a) - (7c) are obtained by using slack variables $x_{k}, y_{k}, z_{k}$ to simplify the original objective function in (6) since the exponential function is an increasing function. Constraint (7d) is obtained from (6a) since all elements are positive. The equivalence between (7) and (6) can be directly shown at the optimum since all the equalities hold in (7a) (7c).

We observe that constraint (7d) is convex because $\frac{1}{1-x}$ is convex in $(0,1)$ and $\operatorname{Tr}(\boldsymbol{X})$ is linear in $\boldsymbol{X}$. Although the objective function and constraints (7c) and (7e) are convex, solving problem (7) is still challenging due to the nonconvexity of constraints (7a), (7b) and (7f). While the rankone constraint can be efficiently handled by the SDR with randomization [27]-[29], dealing with the two first constraints is arduous.

By employing an equivalent form $\beta_{k}^{2}+z_{k}^{2}+2 b \leq \frac{2 y_{k}}{a}+$ $\left(\beta_{k}+z_{k}\right)^{2}$ of (7b), we can rewrite problem (7) as

$$
\begin{aligned}
\underset{\substack{\boldsymbol{W}_{k} \succeq 0, \beta_{k} \\
\max _{k}, y_{k}, z_{k}}}{\operatorname{mamize}} & \frac{E_{\max }}{1-c} \sum_{k=1}^{K}\left(x_{k}-c\right) \\
\text { s.t. } & e^{y_{k}}+1 \leq \frac{1}{x_{k}}, \forall k \\
& \beta_{k}^{2}+z_{k}^{2}+2 b \leq \frac{2 y_{k}}{a}+\left(\beta_{k}+z_{k}\right)^{2}, \\
& (7 \mathrm{c})-(7 \mathrm{f}) .
\end{aligned}
$$

Problem (8) is still difficult due to the non-convexity of constraints (8a) and (8b). Therefore, we propose to solve an equivalent problem as theorem below. 
Theorem 1: The problem (8) is equivalent to the following problem:

$$
\begin{aligned}
& \underset{\substack{\boldsymbol{W}_{k} \succeq 0, \beta_{k} \\
x_{k}, y_{k}, z_{k}, \theta_{k}, \phi_{k}}}{\operatorname{maximize}} \frac{E_{\max }}{1-c} \sum_{k=1}^{K}\left(x_{k}-c\right)+\Theta+\Phi \\
& \text { s.t. }(7 \mathrm{c})-(7 \mathrm{f}) \text {, } \\
& e^{y_{k}}+1 \leq 2 \theta_{k}-\theta_{k}^{2} x_{k}, \forall k, \\
& \beta_{k}^{2}+z_{k}^{2}+2 b \leq \frac{2 y_{k}}{a}+2 \phi_{k}\left(\beta_{k}+z_{k}\right)-\phi_{k}^{2},
\end{aligned}
$$

where $\Theta \triangleq \sum_{k=1}^{K}\left(2 \theta_{k}-x_{k} \theta_{k}^{2}-\frac{1}{x_{k}}\right)$ and $\Phi \triangleq \sum_{k=1}^{K}\left(2 \theta_{k}\left(\beta_{k}+\right.\right.$ $\left.\left.z_{k}\right)-\theta^{2}-\left(\beta_{k}+z_{k}\right)^{2}\right)$.

Proof: See Appendix A.

We observe that problem (9) is a convex optimization problem in $\beta_{k}, x_{k}, z_{k}$ for given $\theta_{k}, \phi_{k}$. In addition, it is also convex in $\theta_{k}$ and $\phi_{k}$ for given $\beta_{k}, x_{k}$ and $z_{k}$. This observation motivates to adopt alternative algorithm to solve the SDR of (8) iteratively. In particular, for given $\theta_{k}, \phi_{k}$, we solve (9) with variable $\beta_{k}, x_{k}, z_{k}$ and get $\left\{\beta_{k}^{\star}, x_{k}^{\star}, z_{k}^{\star}\right\}$. On the other hand, for given $\left\{\beta_{k}^{\star}, x_{k}^{\star}, z_{k}^{\star}\right\}$, we solve (9) with variables $\theta_{k}, \phi_{k}$ and obtain $\theta_{k}^{\star}=\frac{1}{x_{k}^{\star}}$ and $\phi_{k}^{\star}=\beta_{k}^{\star}+z_{k}^{\star}$ (see Appendix A). Details of the proposed algorithm is presented in Algorithm 1. It is noted that problem (10) is convex since the objective function and all constraints are convex.

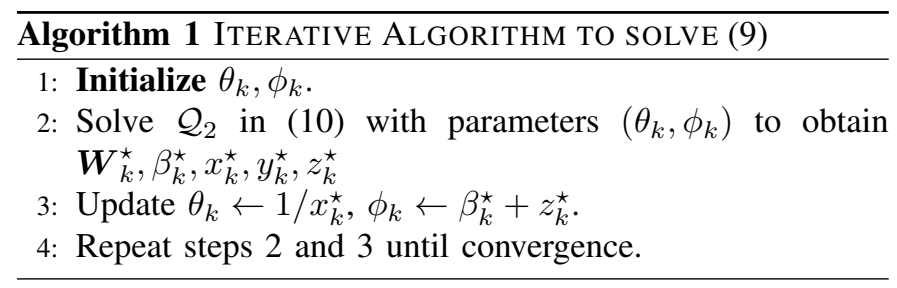

$$
\begin{aligned}
\mathcal{Q}_{2}\left(\theta_{k}, \phi_{k}\right): & \\
\underset{\substack{\boldsymbol{W}_{k} \succeq 0, \beta_{k} \\
x_{k}, y_{k}, z_{k}}}{\max \operatorname{imize}} & \frac{E_{\max }}{1-c} \sum_{k=1}^{K}\left(x_{k}-c\right)+\Theta+\Phi \\
\text { s.t. } & (7 \mathrm{c})-(7 \mathrm{e}),(9 \mathrm{a}),(9 \mathrm{~b}) .
\end{aligned}
$$

Proposition 1 (Convergence): The objective function of (10) in Algorithm 1 decreases over the iterations and the proposed iterative algorithm converges at least to local optimum.

The proof of Proposition 1 can be found by employing a technique similar to the one presented in [30]. Although Proposition 1 does not prove the global optimality, it gives the justification for the iterative algorithm.

Remark 1 (Initialization): Algorithm 1 operates iteratively and hence depends on the initial values of the first-order approximation. For efficient initialization, we first run a convex optimization (11) below

$$
\begin{aligned}
& \underset{\boldsymbol{W}_{k} \succeq 0, \beta_{k}}{\operatorname{maximize}} 1 \quad \text { s.t. } \sum_{k=1}^{K} \operatorname{Tr}\left(\boldsymbol{W}_{k}\right) \leq P_{\text {tot }}, \\
& \frac{\operatorname{Tr}\left(\boldsymbol{H}_{k} \boldsymbol{W}_{k}\right)}{\bar{\eta}_{k}^{R}} \geq \sum_{l \neq k} \operatorname{Tr}\left(\boldsymbol{H}_{k} \boldsymbol{W}_{l}\right)+\frac{\sigma_{A}^{2}}{L_{k}}+\frac{\sigma_{I}^{2}}{L_{k}\left(1-\beta_{k}\right)}, \forall k
\end{aligned}
$$

to obtain the solution $\beta_{k}^{*}, \boldsymbol{W}_{k}^{*}, \forall k$, and then calculate $\theta_{k}=1+e^{a\left(b-\beta_{k}^{*}\left(\sum_{i=1}^{K} L_{k} \operatorname{Tr}\left(\boldsymbol{H}_{k} \boldsymbol{W}_{i}^{*}\right)+\sigma_{A}^{2}\right)\right)}$ and $\phi_{k}=\beta_{k}^{*}+$ $\sum_{i=1}^{K} L_{k} \operatorname{Tr}\left(\boldsymbol{H}_{k} \boldsymbol{W}_{i}^{*}\right)+\sigma_{A}^{2}$.

\section{NUMERICAL RESULTS}

This section presents numerical results to demonstrate the effectiveness of the proposed optimizations. The system operates in the $915 \mathrm{MHz}$ frequency with $B=200 \mathrm{kHz}$ bandwidth [10]. We consider indoor use cases in which the users is uniformly distributed between 0.5 and 5 meters from the BS, e.g., access point. A light-of-sight (LOS) pathloss model is used in which the pathloss in $\mathrm{dB}$ at a distance $d$ in meters from the BS is $31.5+20 \log _{10}(d)-G$, where $G$ is the antenna gain. With $G=10 \mathrm{~dB}$, the actual pathloss $L_{k}$ randomly changes between $25.5 \mathrm{~dB}$ and $45.5 \mathrm{~dB}$. The noise power is $\sigma_{A}^{2}=\sigma_{I}^{2}=-110 \mathrm{dBm}$. Due to the LOS, the wireless channels between the users and the BS are subjected to Rician fading with a Rician factor of $3 \mathrm{~dB}$. Otherwise stated, the BS transmits at $30 \mathrm{dBm}$. The number of BS's antennas is equal to the number of users. The parameters of the $\mathrm{EH}$ model are obtained via curve fitting from the data in [9]. The results are obtained from 100 random user locations and channel realizations. A maximum of 40 iterations is used in our proposed iteration algorithms. The proposed design is compared to i) Ref. [26] which maximizes the minimum received energy, and ii) the so-called Fixed PS scheme which employs the non-linear EH model and spends half of the received power for both energy harvesting and information decoding.

Fig. 2 plots the total harvested energy versus the BS's transmit power while every user is requesting a minimum data rate of $1 \mathrm{Mbps}$. The advantage of the proposed joint optimization is clearly demonstrated via considerable improvement in the total harvested energy compared with the Fixed PS scheme. An approximate gain of $100 \%(3 \mathrm{~dB})$ is observed for the proposed design in the observed transmit power range. Compared to

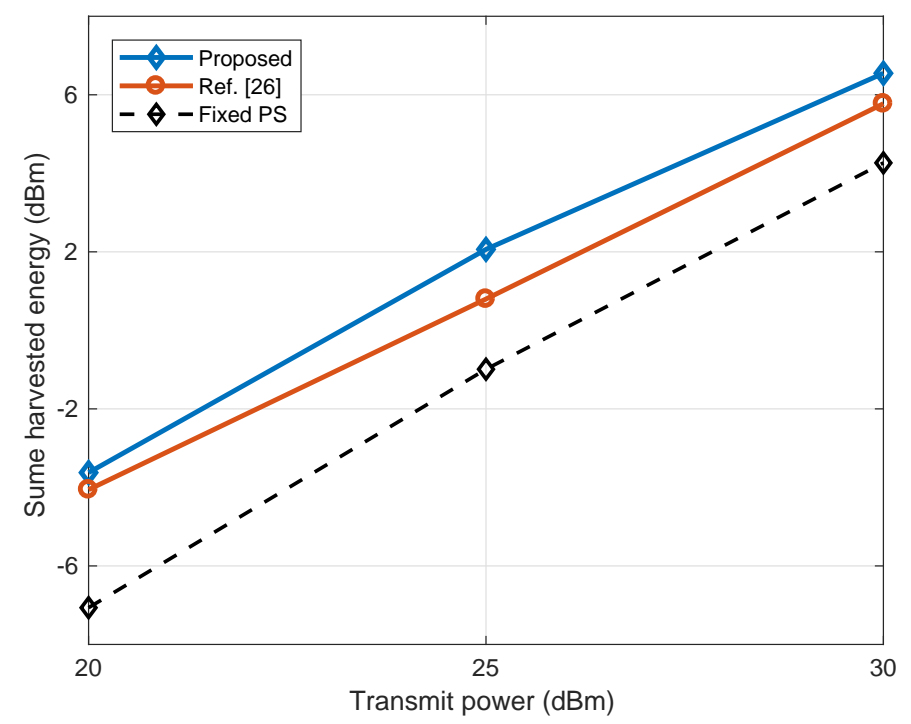

Fig. 2. Total harvested energy versus the transmit power. The BS serves $K=4$ users with 1 Mbps rate requirement. 


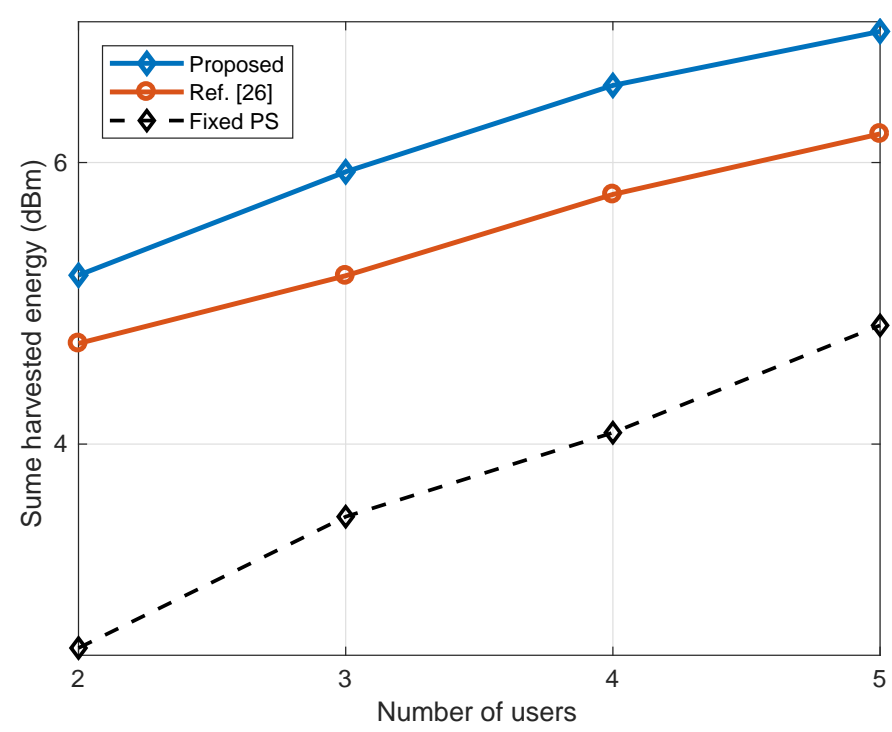

Fig. 4. Total harvested energy versus the number of users $K . P_{t o t}=30$ $\mathrm{dBm}$ and $\eta_{k}^{R}=1 \mathrm{Mbps}, \forall k$.



Fig. 3. Total harvested energy versus the same rate requirement $\eta^{R}=$ $\eta_{k}^{R}, \forall k$. The BS serves $K=4$ users and $P_{\text {tot }}=30 \mathrm{dBm}$.

reference [26], the proposed design harvests $15 \%$ (0.6dB) more efficiently.

Fig. 3 plots the harvested energy as a function of the minimum data rate requirement with the BS's transmit power equal to $30 \mathrm{dBm}$. The figure shows large energy improvement of the proposed scheme compared to both references. In general, the higher the required data rate is, the smaller the harvested energy is. However, the gain of the joint PS and precoding design is preserved for all the data rates. It is also observed that the harvested energy drops at a slower speed than the $\eta^{R}$ 's increasing rate. This is because the data rate is dependent from the received power via a logarithm function.

Fig. 4 presents the total harvested energy for different number of users, where $P_{t o t}=30 \mathrm{dBm}$ and $\eta_{k}^{R}=1 \mathrm{Mbps}, \forall k$. Similar conclusion is obtained that the proposed design obtains a total harvested energy of $80 \%$ and $20 \%$ higher than the fixed PS design and Ref. [26], respectively. An interesting observation is that having more users will result in a larger total harvested energy, although BS's transmit power does not change. This is because having more users will exploit the spatial diversity more efficiently. However, the average harvested energy per user decreases, which is reasonable since the total transmit power is the same.

\section{CONCLUSION}

In this paper, we have investigated the performance of a SWIPT multi-user system with a power splitting mechanism. We developed a framework to jointly optimize the PS factors and precoding vectors under a nonlinear EH model. In particular, we proposed an optimization algorithm to maximize the total harvested energy while guaranteeing the predefined QoS requirements. We showed via numerical results the benefits of the joint design which significantly outperforms the fixed PS and max-min schemes. The outcome of this paper motivates the joint design in MIMO SWIPT systems where the users are equipped with multiple antennas.

\section{ACKNOWLEDGMENT}

This work is supported by the Luxembourg National Research Fund under bilateral project FNR-FNRS InWIP-NET

\section{APPENDIX A}

\section{PROOF OF THEOREM 1}

We will prove that the optimal value of (9) is achievable by (8); the optimal value of (8) is also achievable by (9); and furthermore the optimal solution $\left\{\beta_{k}^{*}\right\}_{k=1}^{K}$ is the same for the two optimizations. To this end, we denote the optimal values of (8) and (9) as $E_{1}^{*}$ and $E_{2}^{*}$, respectively, and will prove $E_{1}^{*}=E_{2}^{*}$. First of all, we note that for an arbitrary positive number $x>0$. We have

$$
2 y-x y^{2} \leq \frac{1}{x}, \forall y \in \mathbb{R}^{+},
$$

where the equality is achieved if and only if $y^{*}=1 / x$. In addition we have $2 x y-y^{2} \leq x^{2}, \forall y \in \mathbb{R}^{+}$, where the equality is achieved if and only if $y^{*}=x$.

\section{A. $S_{1}^{*} \leq S_{2}^{*}$}

First, assume that $\left\{\left\{\beta_{1, k}^{*}, \boldsymbol{W}_{k}^{*}, x_{1, k}^{*}, y_{k}^{*}, z_{1, k}^{*}\right\}_{k=1}^{K}\right\}$ is an optimal solution of (8). Consider a set $\left\{\left\{\beta_{1, k}^{*}, \boldsymbol{W}_{k}^{*}, x_{1, k}^{*}, y_{k}^{*}, z_{1, k}^{*}, \theta_{k}, \phi_{k}\right\}_{k=1}^{K}\right\}$ where $\theta_{k}=\frac{1}{x_{1, k}^{*}}$ and $\phi_{k}=\beta_{1, k}^{*}+z_{1, k}^{*}$. It is straightforward to see that the value $S_{1}^{*}$ is also achieved by (9). Therefore, the optimal value of (9) is $S_{2}^{*} \geq S_{1}^{*}$.

\section{B. $S_{2}^{*} \leq S_{1}^{*}$}

Now assume that $\left\{\beta_{2, k}^{*}, \boldsymbol{W}_{k}^{*}, x_{2, k}^{*}, y_{k}^{*}, z_{2, k}^{*}, \theta_{k}^{*}, \phi_{k}^{*}\right\}_{k=1}^{K}$ is an optimal solution of (9). Consider a new set $\left\{\beta_{2, k}^{*}, \boldsymbol{W}_{k}^{*}, x_{2, k}^{*}, y_{k}^{*}, z_{2, k}^{*}, \frac{1}{x_{2, k}^{*}}, \beta_{2, k}^{*}+z_{2, k}^{*}\right\}_{k=1}^{K}$. We note that 
$\left\{\beta_{2, k}^{*}, \boldsymbol{W}_{k}^{*}, x_{2, k}^{*}, y_{k}^{*}, z_{2, k}^{*}, \frac{1}{x_{2, k}^{*}}, \beta_{2, k}^{*}+z_{2, k}^{*}\right\}_{k=1}^{K}$ satisfies all constraints in (9) since

$$
2 \theta_{k}^{*}-x_{2, k}^{*}\left(\theta_{k}^{*}\right)^{2} \leq \frac{1}{x_{2, k}^{*}}
$$

and

$$
2 \phi_{k}^{*}\left(\beta_{2, k}^{*}+z_{2, k}^{*}\right)-\left(\phi_{k}^{*}\right)^{2} \leq\left(\beta_{2, k}^{*}+z_{2, k}^{*}\right)^{2} .
$$

Furthermore, due to (12) and (13), $\theta_{k}^{*}$ and $\phi_{k}^{*}$ have to be equal to $\frac{1}{x_{2, n}^{*}}$ and $\beta_{2, k}^{*}+z_{2, k}^{*}$, respectively, since otherwise $S_{2}^{*}$ would not be the optimal value of (9). Therefore, from (9) we see that

$$
S_{2}^{*}=\frac{E_{\max }}{1-c} \sum_{k=1}^{K}\left(x_{2, k}^{*}-c\right),
$$

where $\boldsymbol{W}_{k}^{*}, y_{k}^{*}$ satisfies

$$
\begin{gathered}
\sum_{i=1}^{K} L_{k} \operatorname{Tr}\left(\boldsymbol{H}_{k} \boldsymbol{W}_{i}^{*}\right)+\sigma_{A}^{2} \geq z_{2, k}^{*} \\
\frac{\operatorname{Tr}\left(\boldsymbol{H}_{k} \boldsymbol{W}_{k}^{*}\right)}{\bar{\eta}_{k}^{R}} \geq \sum_{l \neq k} \operatorname{Tr}\left(\boldsymbol{H}_{k} \boldsymbol{W}_{l}^{*}\right)+\frac{\sigma_{A}^{2}}{L_{k}}+\frac{\sigma_{I}^{2}}{L_{k}\left(1-\beta_{2, k}^{*}\right)} \\
\exp \left(y_{k}^{*}\right)+1 \leq \frac{1}{x_{2, k}^{*}}, \quad \sum_{k=1}^{K} \operatorname{Tr}\left(\boldsymbol{W}_{k}^{*}\right) \leq P_{t o t} .
\end{gathered}
$$

As the last step, we observe that due to (15)-(17), the $\operatorname{set}\left\{\left\{\beta_{2, k}^{*}, \boldsymbol{W}_{k}^{*}, x_{2, k}^{*}, y_{k}^{*}, z_{2, k}^{*}\right\}_{k=1}^{K}\right\}$ satisfies all constraints of (8). Therefore, we see that $S_{2}^{*}$ is achieved by (8) with the set $\left\{\beta_{k}^{*}, \boldsymbol{W}_{k}^{*}\right\}_{k=1}^{K}$. That leads to $S_{2}^{*} \leq S_{1}^{*}$.

From Section A-A and A-B, we conclude that $S_{1}^{*}=S_{2}^{*}$, which also implies that they have the same optimal solution $\left\{\beta_{k}^{*}, \boldsymbol{W}_{k}^{*}\right\}_{k=1}^{K}$. This concludes the proof for Theorem 1 .

\section{REFERENCES}

[1] Ericsson, "More than 50 billion connected devices ", https://www. ericsson.com/openarticle/mwc-connected-devices_1686565587_c, 2011.

[2] X. Zhou, R. Zhang, and C. K. Ho, "Wireless information and power transfer: Architecture design and rate-energy tradeoff," IEEE Trans. Commun., vol. 61, no. 11, pp. 4754-4767, Nov. 2013.

[3] S. Gautam, T. X. Vu, S. Chatzinotas, and B. Ottersten, "Cache-aided simultaneous wireless information and power transfer (SWIPT) with relay selection," IEEE J. Sel. Areas Commun., vol. 37, no. 1, pp. 187201, Jan. 2019.

[4] Q. Gu, G. Wang, R. Fan, Z. Zhong, K. Yang, and H. Jiang, "Rate-energy tradeoff in simultaneous wireless information and power transfer over fading channels with uncertain distribution," IEEE Trans. Veh. Techno. vol. 67, no. 4, pp. 3663-3668, Apr. 2018.

[5] I. Bang, S. M. Kim, and D. K. Sung, "Adaptive multiuser scheduling for simultaneous wireless information and power transfer in a multicell environment," IEEE Trans. Wireless Commun., vol. 16, no. 11, pp. 74607474, Nov. 2017.

[6] S. Mahama, D. K. P. Asiedu, and K. J. Lee, "Simultaneous wireless information and power transfer for cooperative relay networks with battery," IEEE Access, vol. 5, pp. 13 171-13 178, 2017.

[7] J. Park, B. Clerckx, C. Song, and Y. Wu, "An analysis of the optimum node density for simultaneous wireless information and power transfer in ad hoc networks," IEEE Trans. Veh. Technol., vol. 67, no. 3, pp. 2713-2726, Mar. 2018.

[8] T. Le, K. Mayaram, and T. Fiez, "Efficient far-field radio frequency energy harvesting for passively powered sensor networks," IEEE J. of Solid-State Circuits, vol. 43, no. 5, pp. 1287-1302, May 2008.

[9] J. Guo and X. Zhu, "An improved analytical model for rf-dc conversion efficiency in microwave rectifiers," in Proc. IEEE MTT-S Int. Microw. Symp. Dig., Jun. 2012, pp. 1-3.
[10] E. Boshkovska, D. W. K. Ng, N. Zlatanov, and R. Schober, "Practical non-linear energy harvesting model and resource allocation for SWIPT systems," IEEE Commun. Lett., vol. 19, no. 12, pp. 2082-2085, Dec. 2015.

[11] S. Wang, M. Xia, K. Huang, and Y. Wu, "Wirelessly powered two-way communication with nonlinear energy harvesting model: Rate regions under fixed and mobile relay," IEEE Trans. Wireless Commun., vol. 16, no. 12, pp. 8190-8204, Dec. 2017.

[12] Y. Chen, N. Zhao, and M. Alouini, "Wireless energy harvesting using signals from multiple fading channels," IEEE Trans. Commun., vol. 65, no. 11, pp. 5027-5039, Nov. 2017.

[13] J. Kang, I. Kim, and D. I. Kim, "Wireless information and power transfer: Rate-energy tradeoff for nonlinear energy harvesting," IEEE Trans. Wireless Commun., vol. 17, no. 3, pp. 1966-1981, Mar. 2018.

[14] K. Xiong, B. Wang, and K. J. R. Liu, "Rate-energy region of SWIPT for mimo broadcasting under nonlinear energy harvesting model," IEEE Trans. Wireless Commun., vol. 16, no. 8, pp. 5147-5161, Aug. 2017.

[15] E. Boshkovska, R. Morsi, D. W. K. Ng, and R. Schober, "Power allocation and scheduling for SWIPT systems with non-linear energy harvesting model," in Proc. 2016 IEEE Int. Conf. Commun., May 2016, pp. 1-6.

[16] X. Jia, C. Zhang, and I. Kim, "Worst-case robust beamforming design for wireless powered multirelay multiuser network with a nonlinear EH model," IEEE Trans. Veh. Techno., vol. 68, no. 3, pp. 3038-3042, Mar. 2019.

[17] B. Clerckx, "Waveform and transceiver design for simultaneous wireless information and power transfer," CoRR, vol. abs/1607.05602, 2016. [Online]. Available: http://arxiv.org/abs/1607.05602

[18] B. Clerckx, R. Zhang, R. Schober, D. W. K. Ng, D. I. Kim, and H. V. Poor, "Fundamentals of wireless information and power transfer: From RF energy harvester models to signal and system designs," IEEE J. Sel. Areas Commun., vol. 37, no. 1, pp. 4-33, Jan. 2019.

[19] P. T. D. Ponnimbaduge, D. N. K. Jayakody, S. K. Sharma, S. Chatzinotas, and J. Li, "Simultaneous wireless information and power transfer (SWIPT): Recent advances and future challenges," IEEE Commun. Survey\& Tutorials, vol. 20, no. 1, pp. 264-302, Mar. 2018.

[20] O. T. Demir and T. E. Tuncer, "Maxmin fair resource allocation for SWIPT in multi-group multicast OFDM systems," IEEE Commun. Lett., vol. 21, no. 11, pp. 2508-2511, Nov. 2017.

[21] Q. Shi, L. Liu, W. Xu, and R. Zhang, "Joint transmit beamforming and receive power splitting for MISO SWIPT systems," IEEE Trans. Wireless Commun., vol. 13, no. 6, pp. 3269-3280, Jun. 2014.

[22] M. R. A. Khandaker and K. Wong, "SWIPT in MISO multicasting systems," IEEE Wireless Commun. Lett., vol. 3, no. 3, pp. 277-280, Jun. 2014.

[23] R. Jiang, K. Xiong, P. Fan, S. Zhong, and Z. Zhong, "Optimal beamforming and power splitting design for SWIPT under non-linear energy harvesting model," in Proc. 2017 IEEE Global Commun. Conf., Dec. 2017, pp. $1-6$.

[24] H. Mao, B. Xu, P. Zhu, J. Li, and X. You, "Downlink transmission strategies in power-splitting SWIPT distributed MISO systems," IEEE Access, vol. 6, pp. 52 997-53 005, 2018.

[25] R. Jiang, K. Xiong, P. Fan, Y. Zhang, and Z. Zhong, "Optimal design of SWIPT systems with multiple heterogeneous users under non-linear energy harvesting model," IEEE Access, vol. 5, pp. 1147911489, 2017.

[26] D. Mishra, G. C. Alexandropoulos, and S. De, "Energy sustainable IoT with individual QoS constraints through MISO SWIPT multicasting," IEEE Internet of Things Journal, vol. 5, no. 4, pp. 2856-2867, Aug. 2018.

[27] T. X. Vu, S. Chatzinotas, B. Ottersten, and A. V. Trinh, "Full-duplex enabled mobile edge caching: From distributed to cooperative caching," IEEE Trans. Wireless Commun., vol. xx, pp. 1-1, Dec. 2019.

[28] T. X. Vu, S. Chatzinotas, and B. Ottersten, "Energy-efficient design for edge-caching wireless networks: When is coded-caching beneficial?," in Proc. IEEE Int. Workshop Signal Process. Adv. Wireless Commun., Sapporo, 2017, pp. 1-5.

[29] Z.-Q. Luo, W. K. Ma, A. M. C. So, Y. Ye, and S. Zhang, "Semidefinite relaxation of quadratic optimization problems," IEEE Signal Process. Mag., vol. 27, no. 3, pp. 20-34, Mar. 2010.

[30] H. A. Le Thi, V. N. Huynh, and T. P. Dinh, "DC programming and DCA for general DC programs," in Advanced Computational Methods for Knowledge Engineering. Cham: Springer International Publishing, 2014, pp. 15-35. 